\title{
Perfil das reações adversas imediatas à infusão de quimioterapia em pacientes ambulatoriais em um Hospital Universitário fluminense
}

\author{
Profile of immediate adverse reactions to chemotherapy infusion in outpatients at a University
}

Hospital in the state of Rio de Janeiro

Perfil de reacciones adversas inmediatas a la infusión de quimioterapia en pacientes ambulatorios de un Hospital Universitario de Río de Janeiro

\author{
Maihara da Silva Borges \\ ORCID: https://orcid.org/0000-0002-6893-6919 \\ Hospital do Câncer de Muriaé, Brasil \\ E-mail: maiharaborges61815@gmail.com \\ Marcela Miranda Salles \\ ORCID: https://orcid.org/0000-0002-4339-5546 \\ Hospital Universitário Antônio Pedro, Brasil \\ E-mail: marcela.salles@ebserh.gov.br \\ Ranieri Carvalho Camuzi \\ ORCID: https://orcid.org/0000-0002-5584-8039 \\ Universidade Federal Fluminense, Brasil \\ E-mail: rcamuzi@id.uff.br
}

\begin{abstract}
Resumo
A maioria dos agentes antineoplásicos pode ocasionar reações infusionais, das quais a incidência e o perfil variam conforme as características de cada medicamento, podendo ou não ser imunomediadas. Com o objetivo descrever o perfil das reações adversas imediatas à infusão de quimioterapia em pacientes ambulatoriais, desenvolvemos este estudo exploratório, descritivo, transversal e retrospectivo, a partir registros das notificações das reações adversas realizados pela equipe multiprofissional de março/2018 a março/2019, e dos prontuários dos participantes. As reações adversas imediatas à infusão de quimioterapia foram classificadas quanto à gravidade, de acordo com a Common Terminology Criteria for Adverse Events, e quanto à causalidade usando algoritmo de Naranjo. De um total de 6.832 instalações endovenosas de quimioterapia realizadas no período de estudo, foram registradas 30 reações adversas imediatas, envolvendo 28 pacientes (83\% mulheres), principalmente com Paclitaxel (44\%) e Carboplatina (20\%). Conforme Naranjo, $60 \%$ das reações infusionais foram classificadas como prováveis. O tempo médio para manifestação das reações foi de 40 minutos, sendo a mais rápida com Docetaxel (5 minutos), e a mais demorada com Paclitaxel (180 minutos). A gravidade das reações variou entre leve (64\%) e moderada (30\%). Os segmentos orgânicos mais comprometidos foram respiratório (27\%) e cardiovascular (26\%); e $83 \%$ dos sintomas apresentados estavam previstos nas bases de dados. Com este estudo, foi possível identificar as principais reações imediatas à infusão no grupo em estudo, bem como os quimioterápicos envolvidos com as mesmas, contribuindo para o manejo seguro dos pacientes e no controle eficaz das reações pela equipe multidisciplinar.
\end{abstract}

Palavras-chave: Quimioterapia; Eventos adversos; Farmacovigilância; Segurança do paciente.

\begin{abstract}
Most antineoplastic agents can cause infusion reactions, the incidence and profile of which vary according to the characteristics of each drug, which may or may not be immune-mediated. In order to describe the profile of immediate adverse reactions to chemotherapy infusion in outpatients, we developed this exploratory, descriptive, cross-sectional and retrospective study, based on records of adverse reaction notifications carried out by the multidisciplinary team from March/2018 to March/2019, and the medical records of the participants. Adverse reactions immediate to chemotherapy infusion were ranked for severity according to the Common Terminology Criteria for Adverse Events and for causality using Naranjo's algorithm. Of a total of 6,832 intravenous chemotherapy facilities performed during the study period, 30 immediate adverse reactions were recorded, involving 28 patients (83\% women), mainly with Paclitaxel (44\%) and Carboplatin (20\%). According to Naranjo, 60\% of infusion reactions were classified as probable. The mean time for onset of reactions was 40 minutes, being the fastest with Docetaxel ( 5 minutes) and the longest with Paclitaxel (180 minutes). The severity of reactions ranged from mild (64\%) to moderate $(30 \%)$. The most compromised organic segments were respiratory (27\%) and cardiovascular (26\%); and 83\% of the symptoms
\end{abstract}


presented were predicted in the databases. With this study, it was possible to identify the main immediate reactions to the infusion in the study group, as well as the chemotherapeutic agents involved with them, contributing to the safe management of patients and effective control of reactions by the multidisciplinary team.

Keywords: Chemotherapy; Adverse events; Pharmacovigilance; Patient safety.

\section{Resumen}

La mayoría de los agentes antineoplásicos pueden producir reacciones a la infusión, cuya incidencia y perfil varían según las características de cada fármaco, que pueden ser inmunomediadas o no. Con el fin de describir el perfil de reacciones adversas inmediatas a la infusión de quimioterapia en pacientes ambulatorios, se desarrolló este estudio exploratorio, descriptivo, transversal y retrospectivo, basado en registros de notificaciones de reacciones adversas realizadas por el equipo multidisciplinario de marzo / 2018 a marzo / 2019, y la historia clínica de los participantes. Las reacciones adversas inmediatas a la infusión de quimioterapia se clasificaron por gravedad de acuerdo con los Criterios de terminología común para eventos adversos y por causalidad utilizando el algoritmo de Naranjo. De un total de 6.832 instalaciones de quimioterapia intravenosa realizadas durante el período de estudio, se registraron 30 reacciones adversas inmediatas, involucrando a 28 pacientes (83\% mujeres), principalmente con Paclitaxel (44\%) y Carboplatino (20\%). Según Naranjo, el $60 \%$ de las reacciones a la infusión se clasificaron como probables. El tiempo medio de aparición de reacciones fue de 40 minutos, siendo el más rápido con Docetaxel (5 minutos) y el más largo con Paclitaxel (180 minutos). La gravedad de las reacciones varió de leve (64\%) a moderada (30\%). Los segmentos orgánicos más comprometidos fueron el respiratorio (27\%) y el cardiovascular (26\%); y el 83\% de los síntomas presentados se predijeron en las bases de datos. Con este estudio fue posible identificar las principales reacciones inmediatas a la infusión en el grupo de estudio, así como los agentes quimioterapéuticos involucrados en las mismas, contribuyendo al manejo seguro de los pacientes y al control efectivo de las reacciones por parte del equipo multidisciplinar.

Palabras clave: Quimioterapia; Eventos adversos; Farmacovigilancia; Seguridad del paciente.

\section{Introdução}

O câncer é um nome geral dado a um conjunto de mais de 100 doenças. Inicia-se a partir de uma única célula transformada que não obedece ao regulamento da diferenciação e proliferação celular, crescendo sem respeitar as necessidades do corpo, podendo espalhar-se para tecidos e órgãos vizinhos (Brasil, 2018).

Os tratamentos utilizados para o combate das neoplasias são: cirurgia, radioterapia, hormonioterapia e quimioterapia (Otto, 2002). A quimioterapia é uma das modalidades terapêuticas mais utilizadas e promissoras contra o câncer. É um tratamento sistêmico, que consiste na utilização de compostos químicos, isolados ou em associação, com o objetivo de tratar uma grande variedade de neoplasias malignas (Ribeiro \& Santos, 2015). Por ter uma ação sistêmica, a quimioterapia atinge indiscriminadamente todas as células do organismo, resultando em uma série de reações adversas (Soares et al., 2009).

A reação adversa ao medicamento (RAM) é definida como qualquer resposta prejudicial ou indesejável, não intencional, a um medicamento, que se manifesta após a administração de doses recomendadas para profilaxia, diagnóstico ou tratamento de doenças ( $\mathrm{Pal}$ et al., 2011). As RAMs podem ocorrer de forma imediata, precoce, tardia e ultra tardia, dependentes do tipo de quimioterápico utilizado para o tratamento, dose, tempo de exposição e dos fatores individuais dos pacientes (Ayub et al., 2010).

No que diz respeito ao grau de toxicidade, o Instituto Nacional de Câncer dos Estados Unidos (NCI), no $4^{\circ}$ volume do Common Terminology Criteria for Adverse Events (CTCAE) descreve os eventos adversos, acompanhado de uma escala de gravidade (graus 0 a 5): Grau 0: nenhuma ocorrência de evento adversos; Grau 1: leve; Grau 2: moderada; Grau 3: grave; Grau 4: ameaçador à vida; Grau 5: morte (Cancer Institute, 2009).

Quanto à causalidade das reações adversas, a mesma pode ser estabelecida através de diferentes algoritmos, tais como: Naranjo et al. (1981), Roussel Uclaf Causality Assessment Method (RUCAM), Organização Mundial da Saúde (OMS) e União Européia (WHO, 2010). O algoritmo de Naranjo é o mais comumente utilizado para a determinação da causalidade de um evento, classificando as reações em categorias de probabilidade: definida, provável, possível, condicional ou duvidosa (CFF, 2009). 
O reconhecimento das reações adversas à infusão dos quimioterápicos, assim como da gravidade e das possíveis alternativas para controle e prevenção das mesmas, são alvos de estudo da farmacovigilância, a qual é definida como a ciência e as atividades relativas a detecção, avaliação, compreensão e prevenção de efeitos adversos relacionados a medicamentos (OMS, 2005).

Nesse contexto, tendo em vista a incidência progressiva de casos de câncer nos últimos anos no Brasil, ressalta-se a importância da atuação do farmacêutico na assistência oncológica, junto com a equipe multiprofissional, por ser o profissional responsável pela educação e fornecimento de informações aos pacientes, quanto às possíveis reações adversas aos medicamentos que serão infundidos na quimioterapia; o que pode ser feito para minimizar o aparecimento das mesmas; ou o que fazer quando estas se manifestarem. Dessa forma, o trabalho desenvolvido pelo farmacêutico pode reduzir o agravamento dos sintomas e, consequentemente, o risco de complicação e possíveis internações para os pacientes submetidos ao tratamento quimioterápico (Shamim et al., 2016).

Diante ao exposto, o presente trabalho tem por objetivo descrever o perfil das reações adversas imediatas à infusão de quimioterapia (RAII) em pacientes ambulatoriais de um hospital público da rede sentinela, bem como os fármacos antineoplásicos relacionados às mesmas; descrever os sinais e sintomas apresentados pelos pacientes, e se estão descritos nas respectivas bulas e bases de dados (Micromedex Solutions, Uptodate, artigos científicos); classificar as reações imediatas quanto à gravidade, de acordo com a Common Terminology Criteria for Adverse Events (CTCAE), e quanto à causalidade usando algoritmo de Naranjo.

\section{Metodologia}

Trata-se de um estudo exploratório, descritivo, transversal e retrospectivo (Gil, 2010), realizado no Hospital Universitário Antônio Pedro (HUAP), vinculado à Universidade Federal Fluminense (UFF), sob gestão da Empresa Brasileira de Serviços Hospitalares (EBSERH), localizado em Niterói - RJ.

O HUAP é um hospital geral de médio porte, credenciado à Rede de Hospitais Sentinelas da ANVISA, e referência da Região Metropolitana II do Estado do Rio de Janeiro, que abrange mais de 2 milhões de habitantes e inclui os municípios de Niterói, São Gonçalo, Itaboraí, Rio Bonito, Maricá, Silva Jardim e Tanguá. Classificado como unidade de atendimento de alta complexidade em oncologia (UNACON).

Foram sujeitos do estudo os pacientes do ambulatório de quimioterapia da instituição com diagnóstico de malignidade hematológica ou oncológica, em uso de quimioterapia intravenosa, acometidos pelas reações adversas imediatas à infusão durante o período avaliado.

Os critérios de inclusão adotados foram pacientes a partir de 18 anos, de ambos os sexos com diagnóstico de neoplasia, em tratamento ambulatorial com quimioterápico. Foram excluídos os pacientes que faziam uso de quimioterapia não venosa.

Realizou-se a coleta de dados a partir dos registros das notificações das reações adversas realizados pela equipe multiprofissional à Unidade de Gerência de Riscos Assistenciais do HUAP, no período de março de 2018 a março de 2019. Para obtenção de dados complementares, os pesquisadores acessaram informações contidas no prontuário médico dos participantes da pesquisa.

Como o formulário para notificação de reações adversas disponível na intranet da instituição não dispunha de todas as informações necessárias para posterior avaliação da gravidade e causalidade das mesmas, foi desenvolvido um formulário para registros de RAII de quimioterapia, e utilizado especificamente para coleta destes (Borges,2021). Este formulário foi testado a fim de verificar a sua viabilidade e possíveis falhas, para devidos ajustes. 
Para a determinação da causalidade das reações adversas imediatas em definida, provável, possível ou duvidosa, foi utilizado o Algoritmo de Naranjo e colaboradores. Quanto ao estabelecimento da gravidade da reação em leve, moderada, grave, risco de morte e morte, foi adotada a classificação do National Cancer Institute (NCI) Commom Terminology Criteria for Adverse Events (CTCAE).

Para análise, os dados foram tabulados em planilha eletrônica, a partir da qual as variáveis estudadas foram categorizadas e quantificadas utilizando-se frequência simples.

O estudo foi realizado após a aprovação, pelo Comitê de Ética em Pesquisa da Faculdade de Medicina/HUAP da UFF sob o CAAE 06213518.8.0000.5243, parecer n. $^{\text {o } 3.239 .513 / 2019 . ~}$

\section{Resultados}

\subsection{Incidência de reações adversas relacionadas aos quimioterápicos}

No período compreendido entre março de 2018 a março de 2019, foram realizadas 6.832 instalações endovenosas de quimioterapia no ambulatório do HUAP. Durante essas infusões, 30 pacientes apresentaram reações adversas imediatas à infusão, dos quais 25 (83\%) eram do sexo feminino e 5 (17\%) do sexo masculino, cujas idades variaram entre 33 e 72 anos.

As principais classes de quimioterápicos envolvidas com as reações adversas imediatas à infusão foram Taxanos $(\mathrm{n}=18 ; 60 \%)$ e Platinas $(\mathrm{n}=9 ; 30 \%)$, dentre as quais se destacaram Paclitaxel $(\mathrm{n}=13 ; 44 \%)$ e Carboplatina $(\mathrm{n}=6$; 20\%) respectivamente. As principais reações com Paclitaxel ocorrem entre o $2^{\circ}(\mathrm{n}=5 ; 38,5 \%)$ e $3^{\circ}(\mathrm{n}=5 ; 38,5 \%)$ ciclos de tratamento, já com a Carboplatina as principais reações ocorreram no $6^{\circ}(\mathrm{n}=2 ; 33,3 \%)$ e $7^{\circ}(\mathrm{n}=2 ; 33,3 \%)$ ciclos de tratamento (Tabela 1$)$.

Tabela 1 - Incidência das RAII e os quimioterápicos associados.

\begin{tabular}{|c|c|c|c|c|c|c|c|c|}
\hline Mês & $\begin{array}{l}\text { Paclitaxel } \\
\mathbf{N}(\%)\end{array}$ & $\begin{array}{c}\text { Carboplatina } \\
\mathbf{N}(\%)\end{array}$ & $\begin{array}{c}\text { 5-Fluoruracila } \\
\mathbf{N}(\%)\end{array}$ & $\begin{array}{c}\text { Oxaliplatina } \\
\mathbf{N}(\%)\end{array}$ & $\begin{array}{c}\text { Doxorrubicina } \\
\mathbf{N}(\%)\end{array}$ & $\begin{array}{c}\text { Docetaxel } \\
\mathbf{N}(\%)\end{array}$ & $\begin{array}{l}\text { Rituximabe } \\
\qquad \mathbf{N}(\%)\end{array}$ & Total \\
\hline Mar/18 & $1(3,3)$ & $*$ & $*$ & $*$ & $*$ & $*$ & $*$ & $1(3,3)$ \\
\hline Abr/18 & $3(10,0)$ & $1(3,3)$ & $*$ & $*$ & $*$ & $*$ & $*$ & $4(13,3)$ \\
\hline Mai/18 & $2(6,7)$ & $1(3,3)$ & $*$ & $*$ & $*$ & $*$ & $*$ & $3(10,0)$ \\
\hline Jun/18 & $2(6,7)$ & $1(3,3)$ & $*$ & $1(6,7)$ & $*$ & $*$ & $*$ & $4(13,3)$ \\
\hline Jul/18 & $*$ & $3(10,0)$ & $1(3,3)$ & $*$ & $*$ & $*$ & $*$ & $4(13,3)$ \\
\hline Ago/18 & $*$ & $*$ & $*$ & $*$ & $*$ & $*$ & $*$ & 0 \\
\hline Set/18 & $1(3,3)$ & $*$ & $*$ & $*$ & * & $*$ & $*$ & $1(3,3)$ \\
\hline Out/18 & $*$ & $*$ & $*$ & $1(3,3)$ & $*$ & $*$ & $*$ & $1(3,3)$ \\
\hline Nov/18 & $*$ & $*$ & $*$ & $1(3,3)$ & * & $*$ & $*$ & $1(3,3)$ \\
\hline Dez/18 & $*$ & $*$ & $*$ & $*$ & $*$ & $*$ & $*$ & 0 \\
\hline Jan/19 & * & $*$ & $*$ & $*$ & $1(3,3)$ & $2(6,7)$ & $*$ & $3(10,0)$ \\
\hline Fev/19 & $1(3,3)$ & $*$ & $*$ & $*$ & $*$ & $1(3,3)$ & $1(3,3)$ & $3(6,7)$ \\
\hline Mar/19 & $3(10,0)$ & $*$ & $*$ & $1(3,3)$ & $*$ & $1(3,3)$ & $*$ & $5(16,7)$ \\
\hline Total & $13(43,5)$ & $6(19,9)$ & $1(3,3)$ & $4(13,3)$ & $1(3,3)$ & $4(13,3)$ & $1(3,3)$ & $30(100)$ \\
\hline
\end{tabular}

Fonte: Elaboração própria.

Cabe ressaltar que os principais protocolos quimioterápicos envolvidos com as reações imediatas à infusão foram Paclitaxel + Carboplatina $(n=6 ; 20 \%)$ e Paclitaxel isolado $(n=6 ; 20 \%)$, que foram destinados principalmente ao tratamento de câncer de mama, geniturinário e respiratório. 
3.2 Variáveis quanto ao medicamento que podem impactar no aparecimento de reações adversas e na segurança do paciente

Analisando algumas variáveis importantes quanto ao medicamento que podem impactar na ocorrência de reações adversas a medicamentos e na segurança do paciente, observou-se que 100\% das reações adversas com Paclitaxel (n=13) e Carboplatina $(n=6)$ não ocorreram por sobredose do medicamento e nem por tempo infusional incoerente $(>180 \mathrm{~min}$ e $>60$ min respectivamente). Quanto à realização da Pré-Qt (pré-quimioterapia), nos protocolos contendo Paclitaxel, 23\% foram inadequadas pela ausência de anti-histamínico; 38,5\% delas estavam com tempo entre Pré-Qt e Qt (quimioterapia) adequado (até 1 hora). Nos protocolos contendo Carboplatina, 50\% das Pré-Qt não continham anti-histamínico, apenas corticosteroides; quanto ao tempo entre Pré-Qt e Qt, 66\% dessas reações ocorreram em tempo adequado (até 1 hora) (Figura 1).

Figura 1 - Variáveis quanto ao medicamento.

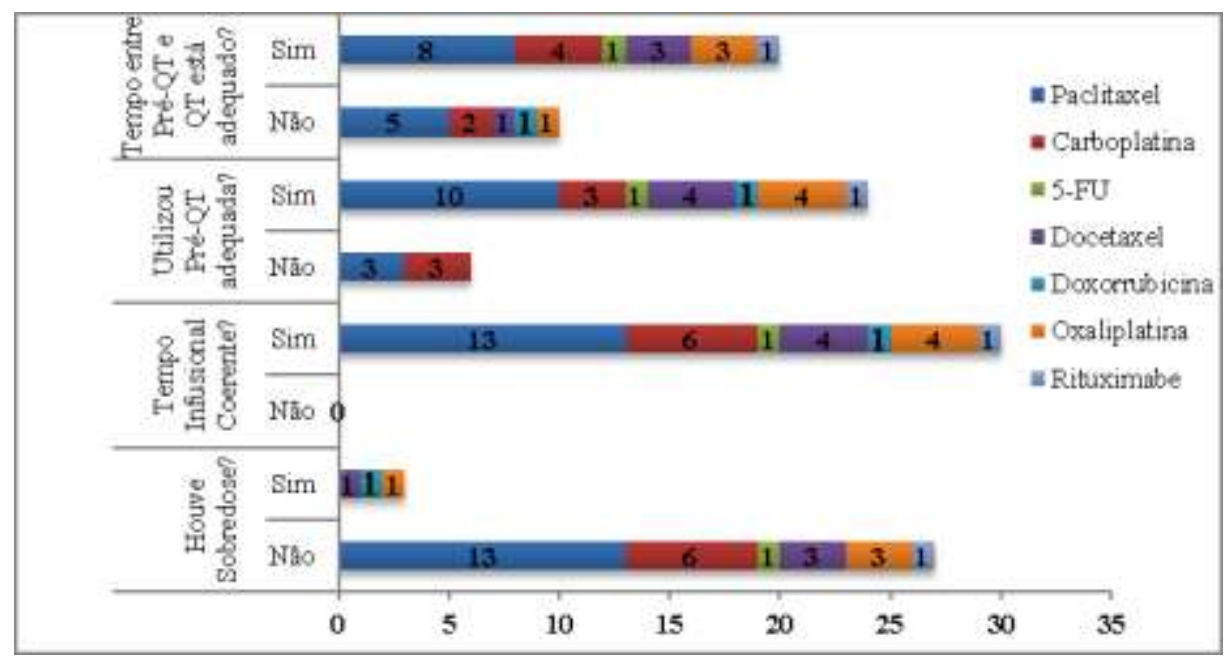

Fonte: Elaboração própria.

\subsection{Tempo entre o início da infusão da quimioterapia e o aparecimento das reações adversas imediatas à infusão}

Verificou-se uma média de 40 minutos entre o tempo decorrido no intervalo do início da infusão da quimioterapia até o início da manifestação de sinais e sintomas. O quimioterápico com manifestação sintomática mais precoce foi o Docetaxel (5 minutos), enquanto aquele com tempo de reação mais tardia foi o Paclitaxel (180 minutos).

\subsection{Incidência dos sintomas apresentados durante as reações adversas imediatas à infusão por segmentos orgânicos} afetados

As reações ocorridas, categorizadas de acordo com CTCAE, envolveram os seguintes órgãos e sistemas: respiratório, cardiovascular, gastrointestinal, neurológico, imunológico, pele, músculo cutâneo e sensorial. De um total de 110 sinais e sintomas apresentados durante as reações, o seguimento respiratório ( $n=30 ; 27 \%)$ foi o mais atingido, especialmente com episódios de dispneia $(n=13 ; 12 \%)$. O seguimento cardiovascular $(n=29 ; 26 \%)$, especialmente com episódios de hipertensão arterial aparece em segundo lugar $(n=7 ; 6,4 \%)$, conforme apresentado na Tabela 2. 
Tabela 2 - Sinais e sintomas caracterizados como reações adversas imediatas à infusão, separados por segmentos orgânicos, a frequência de sua ocorrência.

\begin{tabular}{|c|c|}
\hline SINAIS E SINTOMAS & $\mathbf{N}(\%)$ \\
\hline \multicolumn{2}{|l|}{ SENSORIAL } \\
\hline Sensação de queimação da face & $1(0,9)$ \\
\hline Subtotal & $1(0,9)$ \\
\hline \multicolumn{2}{|l|}{ PELE } \\
\hline Urticária & $2(1,8)$ \\
\hline Prurido & $2(1,8)$ \\
\hline Subtotal & $4(3,6)$ \\
\hline \multicolumn{2}{|l|}{ NEUROLÓGICO } \\
\hline Dificuldade de falar & $2(1,8)$ \\
\hline Dor lombar & $2(1,8)$ \\
\hline Dormência facial & $2(1,8)$ \\
\hline Perda de consciência & $1(0,9)$ \\
\hline Sonolência & $2(1,8)$ \\
\hline Tontura & $2(1,8)$ \\
\hline Tremores generalizados & $2(1,8)$ \\
\hline Subtotal & $13(11,8)$ \\
\hline \multicolumn{2}{|c|}{ MÚSCULO-CUTÂNEO } \\
\hline Hiperemia na palma das mãos & $4(3,6)$ \\
\hline Hiperemia em região torácica & $1(0,9)$ \\
\hline Subtotal & $5(4,5)$ \\
\hline \multicolumn{2}{|l|}{ IMUNOLÓGICO } \\
\hline Rubor facial & $6(5,5)$ \\
\hline Edema periorbitário e de lábio superior & $2(1,8)$ \\
\hline Rash cutâneo & $4(3,6)$ \\
\hline Subtotal & $12(10,9)$ \\
\hline \multicolumn{2}{|c|}{ GASTROINTESTINAL } \\
\hline Diarreia & $2(1,8)$ \\
\hline Dor abdominal & $5(4,5)$ \\
\hline Náuseas & $4(3,6)$ \\
\hline Vômito & $5(4,5)$ \\
\hline Subtotal & $16(14,5)$ \\
\hline \multicolumn{2}{|c|}{ CARDIOVASCULAR } \\
\hline Calor intenso & $5(4,5)$ \\
\hline Cianose & $1(0,9)$ \\
\hline Dor no peito & $2(1,8)$ \\
\hline Hipertensão arterial & $7(6,4)$ \\
\hline Hipotensão & $4(3,6)$ \\
\hline Sudorese & $6(5,5)$ \\
\hline Taquicardia & $4(3,6)$ \\
\hline Subtotal & $29(26,4)$ \\
\hline \multicolumn{2}{|c|}{ RESPIRATÓRIO } \\
\hline Dispneia & $13(11,8)$ \\
\hline Broncoespasmo & $6(5,5)$ \\
\hline Opressão na orofaringe & $7(6,4)$ \\
\hline Tosse seca & $3(2,7)$ \\
\hline Ardência nasal & $1(0,9)$ \\
\hline Subtotal & $30(27,3)$ \\
\hline Total & $110(100)$ \\
\hline
\end{tabular}

Fonte: Elaboração própria.

No que diz respeito à ocorrência das reações entre as classes de quimioterápicos mais envolvidos, destacou-se dispneia $(n=9 ; 17 \%)$, seguido de rubor facial $(n=7 ; 13 \%)$ para os Taxanos, dentre um total de 53 reações. Quanto às Platinas, evidenciou-se broncoespasmo $(n=3 ; 10 \%)$, sudorese $(n=3 ; 10 \%)$ e opressão na orofaringe $(n=3 ; 10 \%)$, dentre o total de 31 reações.

Ao relacionar a sintomatologia apresentada pelos pacientes durante as RAII com os sinais e sintomas previstos e/ou descritos nas bases de dados (Micromedex Solutions, Uptodate, Medscape, Vigiaccess, bula), tem-se que 83\% das RAM estavam descritas. 


\subsection{Causalidade e gravidade das RAM}

Conforme algoritmo de NARANJO, 60\% $(\mathrm{n}=18)$ das reações foram classificadas como causa provável. Das 30 RAII, $19(64 \%)$ se apresentaram como leve conforme categorização do CTCAE; as reações de gravidade moderada somaram um total de $9(30 \%)$, as graves $1(3 \%)$ e ameaçador a vida $1(3 \%)$, conforme relacionado na Tabela 3.

Tabela 3 - Avaliação da Gravidade (CTCAE) e da Causalidade (NARANJO) das RAII.

\begin{tabular}{|c|c|c|c|c|c|c|c|c|}
\hline $\begin{array}{c}\text { Eventos } \\
\text { adversos }\end{array}$ & $\begin{array}{c}\text { Paclitaxel } \\
n=13\end{array}$ & $\begin{array}{c}\text { Carboplatina } \\
n=6\end{array}$ & $\begin{array}{c}\text { Oxaliplatina } \\
n=4\end{array}$ & $\begin{array}{c}\text { Docetaxel } \\
n=4\end{array}$ & $\begin{array}{c}\text { 5-Fluoruracila } \\
n=1\end{array}$ & $\begin{array}{c}\text { Doxorrubicina } \\
n=1\end{array}$ & $\begin{array}{c}\text { Rituximabe } \\
n=1\end{array}$ & Total \\
\hline Graul/Leve & $7(23,3 \%)$ & $4(13,4 \%)$ & $2(6,7 \%)$ & $3(10 \%)$ & $1(3,3 \%)$ & $1(3,3 \%)$ & $1(3,3 \%)$ & $19(64 \%)$ \\
\hline Grau2/Moderada & $5(16,7 \%)$ & $1(3,3 \%)$ & $2(6,7 \%)$ & $1(3,3 \%)$ & --- & --- & --- & $9(30 \%)$ \\
\hline Grau 3/Grave & --- & $1(3,3 \%)$ & --- & --- & & & & $1(3 \%)$ \\
\hline \multirow[t]{2}{*}{$\begin{array}{c}\text { Grau 4/ } \\
\text { Ameaçador à vida } \\
\end{array}$} & $1(3,3 \%)$ & --- & --- & --- & & & & $1(3 \%)$ \\
\hline & & & & & & & & $30(100 \%)$ \\
\hline Provável & $7(23,4 \%)$ & $4(13,4 \%)$ & $3(10 \%)$ & $2(6,7 \%)$ & --- & $1(3,3 \%)$ & $1(3,3 \%)$ & $18(60 \%)$ \\
\hline Possível & $6(20 \%)$ & $2(6,7 \%)$ & $1(3,3 \%)$ & $2(6,7 \%)$ & $1(3,3 \%)$ & --- & --- & $12(40 \%)$ \\
\hline Duvidosa & --- & --- & --- & --- & --- & --- & --- & --- \\
\hline \multirow[t]{2}{*}{ Definida } & $\begin{array}{ll}-- \\
-\end{array}$ & $\begin{array}{ll}-- \\
-\end{array}$ & $\begin{array}{ll}-- \\
-\end{array}$ & --- & $\begin{array}{ll}-- \\
-\end{array}$ & $\begin{array}{ll}-- \\
-\end{array}$ & $\begin{array}{ll}-- \\
-\end{array}$ & $\begin{array}{ll}--- \\
\end{array}$ \\
\hline & & & & & & & & $30(100 \%)$ \\
\hline
\end{tabular}

Fonte: Elaboração própria.

Dentre as reações ocorridas, 90\% exigiram medidas de suporte, que variaram desde hidratação venosa, suspensão temporária ou definitiva da infusão, corticoterapia, até a utilização de oxigenoterapia. Após a aplicação das medidas corretivas, avaliaram-se os desfechos dessas reações, e verificou-se que $70 \%$ dos pacientes obtiveram recuperação completa, enquanto $30 \%$ tiveram prolongamento do tempo de permanência na instituição.

\section{Discussão}

\subsection{Incidência de reações adversas imediatas à infusão relacionadas aos quimioterápicos}

Verificou-se uma incidência de RAII de 0,44. As principais classes de quimioterápicos envolvidas com essas reações foram Taxanos (60\%) e Platinas (30\%), dentre as quais se destacaram Paclitaxel (44\%) e Carboplatina (20\%), respectivamente. No estudo de Bertolazzi et al. (2015), realizado em São José do Rio Preto, foram observados resultados semelhantes, sendo neste caso o Paclitaxel $(30,6 \%)$ e a Oxaliplatina $(23,1 \%)$ os principais quimioterápicos envolvidos com as reações imediatas à infusão. Os Análagos de Platina e os Taxanos estão entre as classes de quimioterápicos mais comumente relacionadas às reações adversas à quimioterapia (Bertolazzi et al., 2015).

O Paclitaxel, quimioterápico associado a maior incidência de reações adversas imediatas à infusão neste estudo (44\%), é um diterpenoide natural extraído da casca do caule da planta alcaloide Taxus brevifolia, e tem sido foco de pesquisa há décadas, devido a seus efeitos terapêuticos únicos e excelente atividade anticancerígena (Chen et al., 2017). Reações de hipersensibilidade são comuns com o Paclitaxel. Os sintomas variam de erupção cutânea, prurido, broncoespasmo, cólicas abdominais, hipotensão e até morte (Weiss et al., 2016). Porém, ainda não foi estabelecido se as reações de hipersensibilidade dependem das características do próprio Taxano, dos seus veículos ou de ambos os componentes (Syrigou et al., 2010).

O mecanismo pelo qual essas reações de hipersensibilidade ao Paclitaxel ocorrem, não é totalmente conhecido, mas acredita-se que possam ser mediadas por efeitos diretos nas células imunológicas ou por outros mecanismos, tais como por ativação do complemento, liberação inespecífica de histamina, produção de óxido nítrico ou liberação de citocinas (Castro et al., 2013). 
De fato, é relatado na literatura que a maioria das reações de hipersensibilidade para os taxanos se desenvolvem nos primeiros 10 a 15 minutos após a infusão (sendo $78 \%$ dos casos nos primeiros 10 minutos), durante a primeira ou segunda infusão, em 95\% dos casos. Apesar de raras, as reações de hipersensibilidade também podem ocorrer durante infusões subsequentes (3\% dos casos), dias ou até semana após a infusão (Syrigou et al., 2010). Os resultados do presente estudo são condizentes, em parte, com a literatura. As principais reações com Paclitaxel ocorreram entre o $2^{\circ}(38,5 \%)$ e o $3^{\circ}(38,5 \%)$ ciclos de tratamento, assim como na maioria dos casos relatados. No que diz respeito ao tempo decorrido entre o intervalo do início da infusão da quimioterapia e o início da manifestação de sinais e sintomas, o quimioterápico com reações mais rápidas foi o Docetaxel (5 minutos), demonstrando que, realmente, com os Taxanos as reações de hipersensibilidade se desenvolvem nos primeiros minutos após a infusão. Porém, o Paclitaxel foi o quimioterápico com manifestação mais tardia, a qual ocorreu após a infusão completa do quimioterápico (180 minutos). A essa exceção vários fatores podem estar associados: características individuais dos pacientes (como história de reações dérmicas leves em ciclos anteriores, exposição repetida a um medicamento da mesma classe química, disfunção respiratória, obesidade, cardiopatias, entre outras comorbidades); características intrínsicas do quimioterápico e fatores extrínsicos, associados aos erros de medicação (Syrigou et al., 2010).

A Carboplatina representa o segundo quimioterápico associado à maior incidência de reações adversas imediatas à infusão neste estudo (20\%). Pertencente à classe dos Agentes Alquilantes, é um quimioterápico análogo das platinas, utilizado para tratar uma ampla variedade de cânceres (Gobel, 2005).

Reações de hipersensibilidade também têm sido associadas ao uso de Carboplatina, provavelmente são reações mediadas por IgE, muito comuns ao longo das reexposições. Reações de hipersensibilidade mediada por anticorpo IgE envolve a ativação de mastócitos, basófilos presentes no sangue periférico, promovendo então liberação de histamina, leucotrienos e prostagladinas. Esse efeito é notado clinicamente com o aparecimento de rash cutâneo, urticária e rubor facial durante o período infusional ou depois de algumas horas, e nos casos mais graves pode ocorrer angioedema, broncoespasmo, hipotensão e disfunção cardíaca (Randall et al., 2013). Postula-se também que a Platina livre acumulada ao longo das infusões pode atuar como alérgeno, assim como suas formas complexadas ao DNA e proteínas causando sensibilização do paciente (Jerzak et al., 2016).

Sliesoraitis e Chikhale (2005) resumiram estudos prévios os quais avaliaram o risco cumulativo de hipersensibilidade em pacientes recebendo Carboplatina em diferentes números de ciclos, sendo que em pacientes que receberam menos de 5 ciclos, houve menos de $1 \%$ de incidência de uma reação de hipersensibilidade. Perceberam que o risco de reações de hipersensibilidade aumenta significativamente no ciclo 6 e continua a aumentar para o ciclo 8 , para um risco cumulativo de 19,5\%. Logo, o risco de desenvolver alguma reação de hipersensibilidade à Carboplatina, é maior quanto maior for o número de ciclos de tratamento. Resultados semelhantes foram encontrados em nosso estudo, uma vez que as principais reações com Carboplatina ocorreram no $6^{\circ}(33,3 \%)$ e $7^{\circ}(33,3 \%)$ ciclo de tratamento.

Por fim, é importante ressaltar que o fato dos principais protocolos quimioterápicos envolvidos com as reações imediatas à infusão terem sido Paclitaxel + Carboplatina e Paclitaxel isolado, pode ter contribuído para que o Paclitaxel tenha sido o principal quimioterápico associado a essas reações.

\subsection{Variáveis quanto ao medicamento que pode impactar no aparecimento de reações adversas e na segurança do paciente}

Neste estudo, considerando os protocolos que continham Paclitaxel e Carboplatina, que foram os principais quimioterápicos envolvidos com as reações adversas imediatas à infusão, verificou-se que 100\% das reações não ocorreram por sobredose do quimioterápico, e nem por tempo ou ordem de infusão incoerente. A escolha por apresentar estes dados 
ocorreu em vista da análise da possível influência dos erros de medicação sobre a incidência de reações adversas imediatas à infusão de quimioterapia, e consequentemente sobre a adesão ao tratamento. No entanto, o impacto do tempo de infusão do Paclitaxel foi avaliado em uma metanálise e não demonstrou diferença no risco de desenvolver reações de hipersensibilidade quando o tratamento foi administrado por 3 ou 24 horas de infusão (Boulanger et al., 2014). Já em relação à Carboplatina, a literatura demonstra que o aumento do tempo de infusão de 3 horas em conjunto com a pré-medicação (em vez da infusão padrão de 60 minutos) pode reduzir o risco de reações de hipersensibilidade (3,4\% vs. 21\%) nos ciclos subsequentes (Lenz, 2007).

A ocorrência de reações de hipersensibilidade pode ser influenciada pela administração de uma pré-medicação adequada. As pré-medicações são geralmente administradas para diminuir a taxa de reações alérgicas e prevenir a ocorrência de reações alérgicas graves durante a infusão do quimioterápico (Wiernik et al., 1987). Atualmente, para hipersensibilidade ao Paclitaxel a pré-medicação padrão consiste em Corticosteroide-Dexametasona, 20mg por via oral (12 h e $6 \mathrm{~h}$ antes da infusão) ou intravenosa (30-60 min antes da infusão); Antagonista H1-Difenidramina 50 mg e Antagonista H2- Ranitidina 50 mg (ou Cimetidina $300 \mathrm{mg}$ ) ambos por via intravenosa 30 a 60 minutos antes do tratamento (Kintzel, 2001).

No presente estudo, apesar da maioria $(23,3 \%)$ das reações imediatas à infusão do Paclitaxel terem sido classificadas como leve, verificou-se que $23 \%$ das pré-medicações para hipersensibilidade, foram inadequadas pela ausência de antihistamínico (Difenidramina). Na verdade, a Difenidramina não era um medicamento padronizado na instituição em questão, o que acabou contribuindo para uma maior incidência de reações adversas à infusão do Paclitaxel. Pode-se observar na Tabela 1, que a partir do mês de agosto de 2018, a incidência de reações adversas com Paclitaxel reduziu o que pode ser explicado também pela padronização da Difenidramina na instituição.

Quanto ao tempo entre a pré-medicação e a infusão do Paclitaxel, observou-se no presente estudo que 38,5\% estavam com tempo adequado, 30 a 60 minutos antes da infusão do Paclitaxel, como preconizado na literatura (Chen et al., 2017). O tempo neste caso é muito importante do ponto de vista farmacocinético, ao passo que, cada medicamento tem um tempo específico para alcançar concentrações plasmáticas mínimas efetivas, e depois serem metabolizados. Sendo assim, se o tempo entre a pré-medicação e a infusão for muito longo pode não haver quantidade suficiente do medicamento na corrente sanguínea que garanta a imunossupressão durante todo o tempo de infusão do quimioterápico, contribuindo para a ocorrência de reações (ICTQ, 2019).

Para hipersensibilidade às Platinas, nenhum regime de pré-medicação padrão/ideal foi estabelecido. Corticosteroides e Antagonistas dos receptores H1 (Difenidramina) + Antagonistas dos receptores H2 (Ranitidina) podem reduzir as taxas de reações adversas à infusão, para alguns pacientes; por exemplo, pacientes ginecológicos com uma história de alergia a outros medicamentos e intervalo sem platina maior que 12 meses. Porém, não há evidências suficientes de que a pré-medicação com corticosteroides ou anti-histamínicos reduza de maneira geral as taxas de reação à infusão(Jerzak et al., 2016). Dessa forma, no presente estudo não foram consideradas como inadequadas as pré-QT sem anti-histamínico para a Carboplatina utilizada de forma isolada. As três reações com Carboplatina consideradas inadequadas quanto à pré-QT, relacionadas na Figura 1, se referem ao protocolo Paclitaxel + Carboplatina, onde o uso de anti-histamínico torna-se necessário devido ao Paclitaxel.

Além da pré-medicação, protocolos de dessensibilização, taxa de infusão mais lenta, testes cutâneos e/ou interrupção do tratamento pode ser uma das estratégias que será seguida após reações infusonais recorrentes, e para os quais não há outras opções de tratamento disponíveis. A estratégia que será seguida depende das características do quimioterápico (Shepherd, 2003). 


\subsection{Incidência dos sintomas apresentados durante as reações adversas imediatas à infusão por segmentos orgânicos afetados}

No presente estudo, as reações ocorridas categorizadas de acordo com CTCAE, atingiram principalmente o seguimento respiratório (27\%), especialmente com episódios de dispneia (43\%). O seguimento cardiovascular (26\%), especialmente com episódios de hipertensão arterial aparece em segundo lugar. No estudo de Bertolazzi et al. (2015), os principais segmentos orgânicos afetados pelas RAII foi o musculocutâneo (52,2\%), especialmente com manifestação de hiperemia facial, enquanto o seguimento respiratório (20,9\%) aparece em segundo lugar, especialmente com episódios de dispneia.

No que diz respeito às principais reações adversas imediatas observadas entre as classes de quimioterápicos mais envolvidos, observaram-se a dispneia (17\%) e o rubor facial (13\%) para os Taxanos. Corroborando estes achados, no estudo de Sidney, Romeu e Pinheiro (2017), no qual as pacientes que apresentaram reações adversas durante os primeiros minutos da infusão do Paclitaxel relataram sintomas relacionados à hipersensibilidade, tais como rubor facial e corporal, dispneia, náusea, hiperemia ocular, calor e tosse seca. De fato, evidências demonstram que com os Taxanos são observadas reações de hipersensibilidade consistentes com uma reação do tipo I, que incluem irritação, erupção cutânea, hipotensão, dispneia e broncoespasmo, observadas minutos após a infusão (Weiss et al., 2016).

Quanto às Platinas, observamos o broncoespasmo (10\%), sudorese (10\%) e opressão na orofaringe (10\%) como as principais reações adversas imediatas. As manifestações clínicas das reações de hipersensibilidade associadas à platina são típicas de uma reação de hipersensibilidade tipo I e incluem urticária, broncoespasmo, erupção cutânea e hipotensão (Lee, Gianos, \& Klaustermeyr, 2009). Polyzos et al. (2001) relataram que a incidência de reações alérgicas em pacientes com câncer de ovário tratadas com carboplatina foi de $16 \%$, principalmente após o $4^{\circ}$ ciclo, sendo a maioria das reações classificadas como leve a moderada. Em nosso estudo, a maioria das reações com a Carboplatina também foi classificada como leve (13,4\%) a moderada $(3,3 \%)$, sendo as principais responsáveis pelo prolongamento do tempo de internação. Isso ocorre, porque geralmente as reações envolvendo a Carboplatina são mais graves e sintomáticas do que aquelas observadas com o Paclitaxel e, por isso, requer mais horas para que os sintomas se resolvam completamente (Muallaoglu et al.,2012).

\subsection{Causalidade e gravidade das RAM}

De acordo com a classificação de NARANJO, 60\% das reações observadas tiveram como causa provável os quimioterápicos. Quanto à gravidade, de acordo com CTCAE, 64\% das reações se manifestaram como grau 1 (leve), sendo essa a reação mais frequente, 30\% como grau 2 (moderada), 3\% grau 3 (grave) e 3\% grau 4 (ameaçador à vida). Resultados parcialmente semelhantes foram observados no estudo de Bertolazzi et al. (2015), onde 79,5\% das reações imediatas tiveram como causa possível os quimioterápicos; e, quanto à gravidade, $50 \%$ foram classificadas como leves.

Em nosso estudo, o Paclitaxel foi o principal quimioterápico associado às RAII. Apesar de ser um medicamento conhecido por maior incidência de reações de hipersensibilidade, ao contrário das platinas, as reações com o Paclitaxel tendem a ser mais uniformes e leves, como pode ser observado na Tabela 2. Dentre as reações classificadas como leves, as mais frequentes foram com Paclitaxel (23,3\%); e, quanto à causalidade, conforme algoritmo de Naranjo, considerou-se provável. Esse acaba sendo um resultado questionável, pois o Naranjo é um algoritmo que, como qualquer outro, tem suas limitações/falhas, exigindo maior conhecimento farmacológico por parte do avaliador e/ou interesse em encontrar as informações necessárias, o que também pode contribuir para uma mensuração incorreta do desfecho (Menezes \& Chinchilla, 2011). 
Neste estudo, $90 \%$ das reações exigiram medidas de suporte, que variaram desde a hidratação venosa, a suspensão temporária ou definitiva do quimioterápico até a oxigenoterapia. Em $70 \%$ dos pacientes que receberam alguma medida corretiva, a recuperação foi completa e imediata. Provavelmente, esse resultado foi associado ao fato de que as reações relacionadas ao Paclitaxel (44\%) geralmente se resolvem rapidamente após a descontinuação da infusão, e não requer, na maioria das vezes, a substituição por outro medicamento.

\section{Conclusão}

Com o presente estudo foi possível levantar o perfil das reações adversas imediatas à infusão de quimioterapia em pacientes ambulatoriais da instituição. Esse trabalho impulsionou melhorias no processo de notificação, demonstrou a importância da padronização do anti-histamínico Difenidramina nos protocolos com Paclitaxel, colaborando para reduzir a incidência de novas RAII. Além disso, evidenciou a necessidade de inserção do farmacêutico clínico realizando, entre outras atividades, a farmacovigilância.

As reações adversas identificadas foram majoritariamente classificadas como leves. E a maioria das reações notificadas envolveram os Taxanos e Análogos de Platina. O Paclitaxel foi o principal quimioterápico associado às reações adversas imediatas à infusão, cujas reações de hipersensibilidade apesar de classificadas como leves foram desencadeadas tanto imediatamente à infusão, quanto próximo do término, afetando principalmente o sistema respiratório, especialmente com episódios de dispneia.

Estudos futuros poderiam manter o monitoramento das reações adversas à quimioterapia venosa de forma prospectiva, verificando a manutenção dos benefícios ora identificados, bem como gerando mais informações, durante maior tempo e com uma população ainda mais ampliada.

\section{Agradecimentos}

Ao Hospital Universitário Antônio Pedro (HUAP); ao Programa de Pós-graduação Lato Sensu Residência Multiprofissional em Saúde do HUAP; ao Ministério da Saúde (MS); ao Ministério da Educação (MEC); à Universidade Federal Fluminense (UFF).

\section{Referências}

Ayub, M. N., da Silva, D., Martinbiancho, J. K., \& Dal-Pizzol, T. S. (2010). Adverse Drug Reactions in Patients Hospitalized in the Intensive Care Unit of a University Hospital in Southern Brazil. Am. J. Pharm, 29(5), 688-693.

Bertolazzi, L. G., Lanza, M. V. de C., Bitencourt, E. C., Canille, R. M. da S., Pereira, L. P. de S., Oliveira, K. A. de, \& Fernandez, F. L. C. (2015). Incidência E Caracterização De Reações Adversas Imediatas À Infusão De Quimioterápicos Em Hospital Sentinela. Arquivos de Ciências Da Saúde, 22(3), 84-90. https://doi.org/10.17696/2318-3691.22.3.2015.107.

Borges, M. da S., Salles, M.M., \& Camuzi, R.C. (2021). Incidência e caracterização das reações adversas imediatas e tardias à infusão de quimioterapia em pacientes ambulatoriais de um hospital sentinela. Trabalho de Pós-Graduação em oncologia, Universidade Federal Fluminense, Rio de Janeiro, Brasil.

Boulanger, J., Boursiquot, J. N., Cournoyer Md, G., Lemieux, J., Almanric, || K, Msc, B., \& Guay Bpharm Msc, \# M P. (2014). Management Of Hypersensitivity To Chemotherapy Management of hypersensitivity to platinum-and taxane-based chemotherapy: cepo review and clinical recommendations. Current OnCOlOgy, 21(4). https://doi.org/10.3747/co.21.1966

Brasil. (2018). Ministério da Saúde. ABC do câncer: abordagens básicas para o controle do câncer. Instituto Nacional de Câncer José Alencar Gomes da Silva. INCA:2018.https://www.inca.gov.br/sites/ufu.sti.inca.local/files//media/document//livro-abc-4-edicao.pdf

Cancer Institute, N. (2009). Common Terminology Criteria for Adverse Events (CTCAE) Version 4.0. http://www.meddramsso.com

Castro, I. J., Pons Busom, M., Ballesteros Cabañas, G. I., Ramió Montero, E., Álvarez Méndez, A., de Castro Chivato, R., \& Centelles Ruiz, M. (2013). Estudio de las reacciones adversas relacionadas con la infusión de paclitaxel y docetaxel. Farmacia Hospitalaria, 37(2), 88-94. https://doi.org/10.7399/FH.2013.37.2.374 
Research, Society and Development, v. 10, n. 15, e416101523009, 2021 (CC BY 4.0) | ISSN 2525-3409 | DOI: http://dx.doi.org/10.33448/rsd-v10i15.23009

Chen, F.-C., Wang, L.-H., Zheng, X.-Y., Zhang, X.-M., Zhang, J., \& Li, L.-J. (2017). Meta-analysis of the effects of oral and intravenous dexamethasone premedication in the prevention of paclitaxel-induced allergic reactions. Oncotarget, 8(12), 19236-19243. www.impactjournals.com/oncotarget/

Conselho, F.de F. (2009). Farmácia hospitalar. Farmacêutico em oncologia: interfaces administrativas e técnicas. Instituto do Câncer do Ceará. https://pt.scribd.com/document/72131094/Farmaceutico-em-Oncologia-Interfaces-Administrativas-e-Clinicas

Gil, A. C. (2010). Como elaborar projetos de pesquisa (5 ed). Atlas.

Gobel, B. H. (2005). Chemotherapy-induced hypersensitivity reactions. Oncology Nursing Forum, 32(5), 1027-1035. https://doi.org/10.1188/05.ONF.10271035

Instituto, de C.T.Q. (2019) Farmacocinética clínica e farmacodinâmica. https://ictq.com.br/varejo-farmaceutico/838-farmacocinetica-clinica-efarmacodinamica

Jerzak, K. J., Deghan Manshadi, S., Ng, P., Maganti, M., Mccuaig, J. M., Bulter, M., Oza, A., \& Mackay, H. J. (2016). Prevention of carboplatin-induced hypersensitivity reactions in women with ovarian cancer. https://doi.org/10.1177/1078155216679028

Kintzel, P.E. (2001). Prophylaxis for Paclitaxel hypersensitivity reactions. Annals of Pharmacotherapy,35(9),1114-1117. 10.13145/aph.10287

Lee, C., Gianos, M., \& Klaustermeyer, W. B. (2009). Diagnosis and management of hypersensitivity reactions related to common cancer chemotherapy agents. In Annals of Allergy, Asthma and Immunology 102(3), 179-187. American College of Allergy, Asthma and Immunology. https://doi.org/10.1016/S1081-1206(10)60078-6

Lenz, H.-J. (2007). Management and Preparedness for Infusion and Hypersensitivity Reactions. https://doi.org/10.1634/theoncologist.12-5-601

Menezes, T. V. N., \& Chinchila, I. (2011). Determinação de causalidade em processos de farmacovigilância. Trabalho de Pós-Graduação em Vigilância Sanitária, Pontífice Universidade Católica de Goiás, Goiás, Brasil 2011

Muallaoglu, S., Disel, U., Mertsoylu, H., Besen, A., Karadeniz, C., Sumbul, A. T., Abali, H., \& Ozyilkan, O. (2013). Acute infusion reactions to chemotherapeutic drugs: A single institute experience. J Buon,18, 261-267.

Naranjo, C.A., Busto, U., Sellers, E.M., Sandor, P., Ruiz, I., Roberts, E.A., Janecek, E., Domecq, C., \& Greenblett, D.J. (1981). A method for estimating the probability of adverse drug reactions. Clin Pharmacol Ther, 30(2):239-245.

Organização Mundial Da Saúde. (2005). Monitorização da segurança de medicamentos: diretrizes para criação e funcionamento de um centro de farmacovigilância. Brasília: Organização Pan-Americana da Saúde, 2005. https://bvsms.saude.gov.br/bvs/publicacoes/seguranca.pdf

Otto, S. (2002). Oncologia. Reichmann \& Affonso Editores.

Pal, S., Dodoo, A., Mantel, A., \& Olsson, S. (2011). The World Medicines Situation 2011 Pharmacovigilance And Safety Of Medicines.

Polyzos, A., Tsavaris, N., Kosmasa, C., Arnaouti, T., Kalahanis, N., Tsigris, C., Giannopoulos, A., Karatzas, G., Giannikos, L., \& Sfikakis, P. P. (2001) Hypersensitivity reactions to carboplatin administration are common but not always severe: A 10-year experience. Oncology, 61,129-133.

Randall, J. M., Bharne, A. A., \& Bazhenova, L. A. (2013). Hypersensitivity reactions to carboplatin and cisplatin in non-small cell lung cancer. Journal of Thoracic Disease, 5(2). https://doi.org/10.3978/j.issn.2072-1439.2012.06.12

Ribeiro, T. dos S., \& Santos, V. O. (2015). Segurança do Paciente na Administração de Quimioterapia Antineoplásica: uma Revisão Integrativa. Revista Brasileira de Cancerologia, 61(2), 145-153. https://doi.org/10.32635/2176-9745.RBC.2015V61N2.554

Shamim, S., Sharib, S. M., Malhi, S.M., Muntaha, S., Raza, H., Ata, S., Farooq, A. S., \& Hussain, U. M. (2016). Adverse drug reactions (ADRS) reporting: awareness and reasons of under-reporting among health care professionals, a challenge for pharmacists. SpringerPlus, 5(1). https://doi.org/10.1186/S40064016-3337-4

Shepherd, G. M. (2003). Hypersensitivity reactions to chemotherapeutic drugs. Clinical Reviews in Allergy \& Immunology, 24(3), 253-262. https://doi.org/10.1385/CRIAI:24:3:253

Sidney, K. M. M., Romeu, G. A., \& Pinheiro, C. (2017). Estudo de reações adversas ao paclitaxel em pacientes com câncer de mama em um hospital de referência. Revista Brasileira de Farmácia Hospitalar, 8 (1), 37-42.

Soares, L.C., Antonacci, M.H., Santana, M. da G \& Schwartz, E. (2009). A quimioterapia e seus efeitos adversos: relato de clientes oncológicos. Cogityare Enferm, 14, 714-719.

Syrigou, E., Triantafyllou, O., Makrilia, N., Kaklamanos, I., Kotanidou, A., Manolopoulos, L., \& Syrigos, K. (2010). Acute Hypersensitivity Reactions to Chemotherapy Agents: An Overview. Inflammation \& Allergy-Drug Targets, 9, 206-213.

Weiss, R. B., Donehower, R. C., Wiernik, P. H., Ohnuma, T., Gralla, R. J., Trump, D. L., Jr, J. R. B., Echo, D. A. van, Hoff, D. D. von, \& Leyland-Jones, B. (2016). Hypersensitivity reactions from taxol. Https://Doi.Org/10.1200/JCO.1990.8.7.1263, 8(7), 1263-1268. https://doi.org/10.1200/JCO.1990.8.7.1263

Wiernik, P. H., Schwartz, E. L., Strauman, J. J., Dutcher, J. P., Lipton, R. B., \& Paietta, E. (1987). Phase I Clinical and Pharmacokinetic Study of Taxol1. Cancer Research, 47, 2486-2493.

World Health Organization. (2010). Uppsala monitoring centre. Programme for international drug monitoring. http://www.whoumc.org/DynPag e.aspx id $=13140 \& m n=1514 \# 3$ 